\title{
Quantifying and Overcoming the Effect of Distraction on Cognitive Load in a Brain-Computer Interface
}

\author{
Zahra Emami ${ }^{1}$, Tom Chau ${ }^{1,2}$ \\ ${ }^{1}$ Institute of Biomaterials and Biomedical Engineering, University of Toronto \\ 164 College Street, Toronto, Canada \\ zahra.emami@mail.utoronto.ca; tom.chau@utoronto.ca \\ ${ }^{2}$ Bloorview Research Institute, Holland Bloorview Kids Rehabilitation Hospital \\ 150 Kilgour Road, Toronto, Canada \\ tchau@hollandbloorview.ca
}

\section{Extended Abstract}

A Brain-Computer Interface (BCI) is a technology that allows its user to operate a device or application by means of cognitive activity. Because they do not require any motor input, BCIs have significant applications for individuals with severely impaired motor control. While the ultimate setting for the use of BCIs are real-world environments such as clinics and homes, this technology has largely been examined in laboratory conditions [1]. However, laboratory conditions aren't often comparable to real-world environments. The effects of certain variables present in the real-world, which may not be present in the lab, may impede the successful use of BCIs by the end-user. One environmental factor whose influence on BCIs has yet to be systematically examined is a distraction. Salient task-irrelevant information has the potential to capture attention, and by consuming a limited set of cognitive resources, places an increased load on the cognitive processing of the task at hand. The resulting distraction can therefore diminish task performance. The purpose of the current study is twofold: (i) to determine the effect of distractions in BCIs, given the effect on the user's cognitive load, and (ii) to examine whether performance amid distractions can be improved through techniques that moderate cognitive load. Twenty-five able-bodied participants competed training to achieve motor imagery-guided BCI control in both offline and online settings. Electroencephalography (EEG) is used to measure the distinctive changes in activity of the sensorimotor rhythm that occur in accordance with the imagination of left and right hand movement. Those participants that achieve satisfactory control of the motor-imagery EEG-BCI then undergo the BCI task in conditions of no distraction, visual distraction, and distraction along with cognitive load-mediating techniques. These cognitive strategies will involve modulating the perceptual and working memory load of the interface in order to decrease the overall cognitive load entailed by the motor imagery task. BCI performance during the different conditions is determined based on the classification accuracy of the BCI, while cognitive load is assessed through both the NASA-Task Load Index [2] and the index of frontal theta and central-parietal alpha power [3], as subjective and objective measures of task difficulty. Preliminary results show that the majority of participants are able to achieve above-chance performance in using motor imagery to control a BCI, with an average classification value of 0.71 . Performance is expected to be diminished during trials with distractors, in accordance with an expected increase in cognitive load. Early results suggest that the distractors may not only have a local effect at a single trial level, but in some participants, may have a global effect, impacting overall BCI performance throughout the session. Reducing the cognitive load placed by the task, by manipulating task difficulty, is expected to rescue performance [4]. Accounting for the effect of distractions in BCIs can help facilitate the effective translation of the technology from the lab to the home.

\section{References}

[1] H. J. Hwang, S. Kim, S. Choi and C. H. Im, "EEG-based brain-computer interfaces: A thorough literature survey," International Journal of Human-Computer Interaction, vol. 29, no. 12, pp. 814-826, 2013.

[2] S.G. Hart, "NASA-Task Load Index (NASA-TLX); 20 Years Later," in Proceedings of the Human Factors and Ergonomics Society Annual Meeting, Santa Monica, CA, 2006, vol. 50, no. 9, pp. 904-904. 
[3] P. Antonenko, F. Pass, R. Grabner and T. Van Gog, "Using electroencephalography to measure cognitive load," Educational Psychology Review, vol. 22, no. 4, pp. 425-438, 2010.

[4] N. Lavie and Y. Tsal, "Load theory of selective attention and cognitive control," Journal of Experimental Psychology: General, vol. 133, no. 3, pp. 339-354, 2004. 